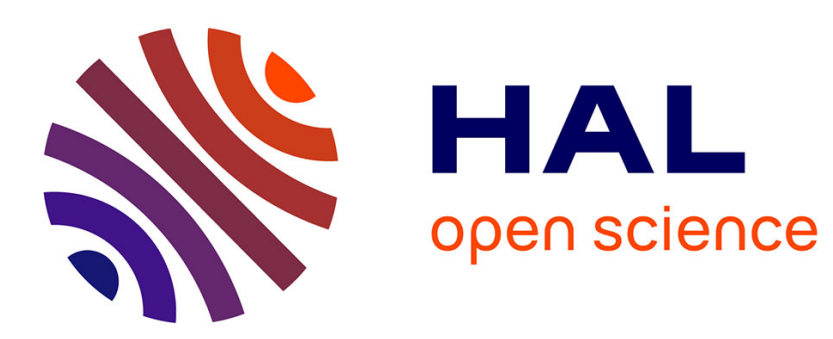

\title{
Frictional Resistance within the Wake of Frictional Rupture Fronts
}

\author{
Ilya Svetlizky, Elsa Bayart, Gil Cohen, Jay Fineberg
}

\section{To cite this version:}

Ilya Svetlizky, Elsa Bayart, Gil Cohen, Jay Fineberg. Frictional Resistance within the Wake of Frictional Rupture Fronts. Physical Review Letters, 2017, 118 (23), 10.1103/physrevlett.118.234301. hal-03025985

\section{HAL Id: hal-03025985 \\ https://hal.science/hal-03025985}

Submitted on 26 Nov 2020

HAL is a multi-disciplinary open access archive for the deposit and dissemination of scientific research documents, whether they are published or not. The documents may come from teaching and research institutions in France or abroad, or from public or private research centers.
L'archive ouverte pluridisciplinaire HAL, est destinée au dépôt et à la diffusion de documents scientifiques de niveau recherche, publiés ou non, émanant des établissements d'enseignement et de recherche français ou étrangers, des laboratoires publics ou privés. 


\title{
Frictional Resistance within the Wake of Frictional Rupture Fronts
}

\author{
Ilya Svetlizky, Elsa Bayart, Gil Cohen, and Jay Fineberg \\ The Racah Institute of Physics, The Hebrew University of Jerusalem, Jerusalem 91904, Israel
}

(Received 15 March 2017; published 8 June 2017)

\begin{abstract}
Frictional resistance to slip, $\tau$, is determined by the real area of contact, $A$, and the shear strength of the contacts forming the frictional interface. We perform simultaneous high-speed local measurements of $\tau$ and $A$ at the tail of propagating rupture fronts. Rate dependence is investigated over 2 orders of magnitude of local slip velocities which reach up to $\sim 1 \mathrm{~m} / \mathrm{s}$. A critical slip velocity is observed that signifies a transition in the frictional behavior: enhanced velocity weakening of $A$ and $\tau$. These measurements enable us to infer the contact shear strength, an otherwise elusive quantity, and show that the contact shear strength persistently increases with slip rate. This, surprisingly, contrasts with expected contact softening at the high temperatures induced by rapid sliding.
\end{abstract}

DOI: 10.1103/PhysRevLett.118.234301

The real area of contact formed by two rough surfaces is substantially smaller than the ostensible contact area, as it is composed of a myriad of discrete microscopic contacts $[1,2]$. The pressure at these contacts is therefore huge, often reaching material yield stresses. The mechanical properties of the frictional interface consequently differ significantly from those of the elastic bulk material. Although critically important, direct measurement of interface properties is elusive, as they are hidden within the microscopically thin frictional interface. Two of these, the real area of contact and the contact shear strength, determine the frictional resistance to sliding [3]. These are crucial for our fundamental understanding of friction and directly impact applications ranging from the sliding of everyday macroscopic objects to the relative motion of tectonic plates.

The frictional resistance of sliding bodies is commonly represented by an effective single degree of freedom, the "dynamic friction coefficient" in experiments where the sliding velocities, $v$, are externally imposed with values $0.01 \mu \mathrm{m} / \mathrm{s}<v<\sim 1 \mathrm{~m} / \mathrm{s}$. In such experiments, friction commonly weakens logarithmically with increasing $v$ [3-6] for slow velocities. This weakening has been interpreted as thermally activated creep [3,7-9], that often crosses over to velocity strengthening at higher slip rates [10-12]. At extreme values $(v>0.1 \mathrm{~m} / \mathrm{s})$ substantial weakening occurs that often (but not always [13]) is attributed to significant temperature increase [14-18]. Extreme local values of $v$ occur naturally in the transition to frictional motion, that occurs via dynamically propagating fronts that rupture the contacts forming the frictional interface [19]. These ruptures generate local slip velocities whose values often surpass the most rapid imposed $v$ [20-26]. The stresses and material velocities at the tip of these rupture fronts are described by the singular solutions of linear elastic fracture mechanics, LEFM, that describe brittle shear cracks $[22,24,27]$. The rapid propagation of these singularities has impeded experimental study of frictional resistance evolution.

Here we experimentally study the frictional resistance (the local dynamic friction coefficient) in the wake of propagating rupture fronts whose propagation velocities range from a small fraction of the Rayleigh wave speed, $C_{R}$, to values that either asymptotically approach $C_{R}$ or surpass the shear wave speed, $C_{S}$ to reach the longitudinal speed of sound, $C_{L}$. By combining high-speed measurements of the stresses adjacent to the frictional interface, the real area of contact and sliding velocities we trace the interrelation of these dynamic quantities. We find that, surprisingly, the velocity dependence of the local frictional resistance is both nontrivial and implies that contacts persistently strengthen-even at extreme values of $v$.

We study the interface strength of contacting poly (methylmethacrylate) (PMMA) plates $\left(\rho \approx 1,170 \mathrm{~kg} / \mathrm{m}^{3}\right)$. The $x, y, z$ dimensions of the upper plate were 200,100, $5.5 \mathrm{~mm}$ [Fig. 1(a)]. Lower plates were either thin (240, 100, $5.5 \mathrm{~mm}$ ) or thick $(300,30,30 \mathrm{~mm})$. Rayleigh, shear, and longitudinal wave speeds are, respectively, $C_{R} \approx 1237 \mathrm{~m} / \mathrm{s}$, $C_{S} \approx 1345 \mathrm{~m} / \mathrm{s}$, and $C_{L} \approx 2333 \mathrm{~m} / \mathrm{s}$ (plane stress) [24]. The system is approximately $2 \mathrm{D}$ with a quasi $1 \mathrm{D}$ frictional interface, as the $5.5 \mathrm{~mm}$ interface width is considerably smaller than other system dimensions. The contacting surfaces were cleaned by distilled water and isopropyl alcohol and then dried for about $2 \mathrm{~h}$ [28]. A high speed camera (580000 frames/s) was used to visualize the dynamic changes in the real area of contact, $A(x, t)$, via a method of total internal reflection $[19,24,32]$. The entire $200 \mathrm{~mm} \times 5.5 \mathrm{~mm}$ frictional interface was mapped to $1280 \times 8$ pixels; each pixel represented the sum value of a large ensemble of discrete contacts. The three components of the 2D strain tensor, $\varepsilon_{i j}(x, t)$, were continuously and simultaneously measured every $1 \mu \mathrm{s}$ at 16-19 spatial locations at $y \approx 3.5 \mathrm{~mm}$ above the interface [Fig. 1(a)]. The two plates were carefully aligned and pressed together 

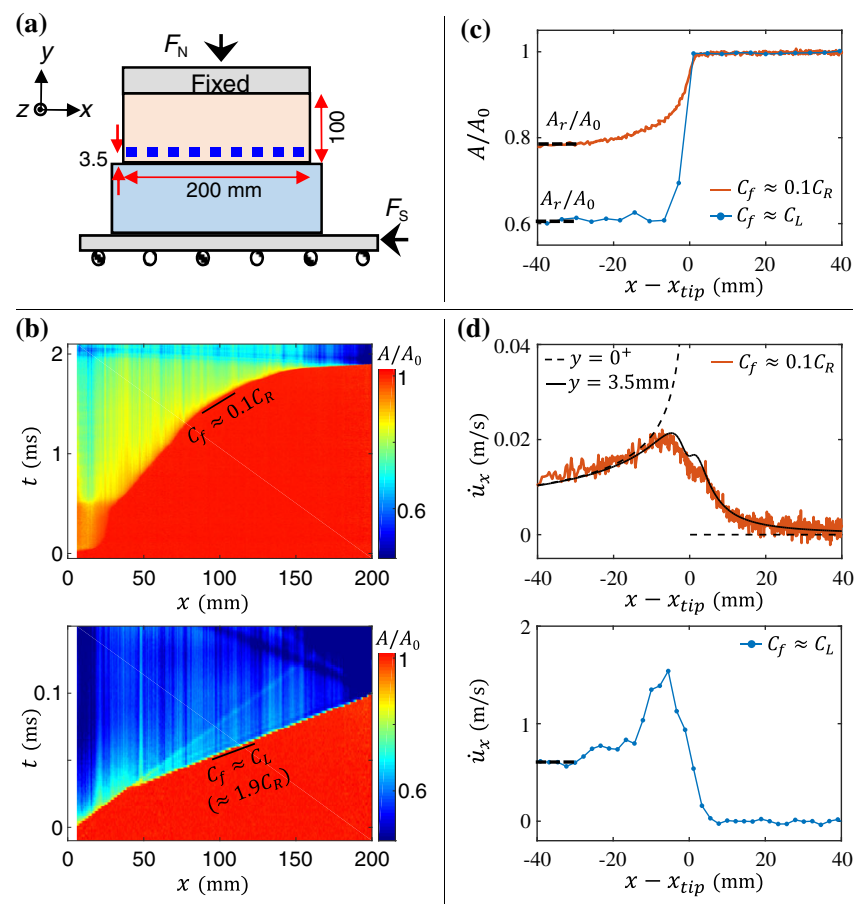

FIG. 1. Evolution of the real area of contact and material velocities during rupture front propagation. (a) Two PMMA blocks are used in a stick slip friction experiment. Rosette strain gauges, mounted within the upper plate $\sim 3.5 \mathrm{~mm}$ above the frictional interface, measure all components of the 2D strain tensor every $1 \mu \mathrm{s}$. (b) The evolution of $A(x, t)$ for typical slow (top) and supershear (bottom) rupture fronts. The fronts nucleate at $x \approx 0$ and reduce $A(x, t)$ in their wake. (c) Reduction of $A / A_{0}$ with local rupture velocities $C_{f} \approx 0.1 C_{R}$ (orange line) and $C_{f} \approx$ $C_{L}$ (blue line). $A_{r}$ denotes the residual area of contact at the tail of the propagating rupture front. (d) Material velocities, $\dot{u}_{x}$ at $y \approx 3.5 \mathrm{~mm}$, inferred from measured $\varepsilon_{x x}$ [24]. (top) Slowly propagating rupture, with LEFM prediction (solid line). $\Gamma \approx 2.5 \mathrm{~J} / \mathrm{m}^{2}$, the fracture energy, is the sole free parameter [24]. Dashed line is the singular velocity, $\dot{u}_{x}\left(y=0^{+}\right)$, predicted by LEFM. At $x-x_{\text {tip }}<-20 \mathrm{~mm}$ measured $\dot{u}_{x}(y=3.5 \mathrm{~mm})$ correspond to $\dot{u}_{x}\left(y=0^{+}\right)$. (bottom) $\dot{u}_{x}$ for a supershear rupture. (c),(d) Rupture tip position, $x_{\text {tip }}=110 \mathrm{~mm}$. Lower values of $A_{r}$ correspond to faster $\dot{u}_{x}$.

by an external normal force, $F_{N} \approx 5,500 N$ ( $5 \mathrm{MPa}$ of nominal pressure) [Fig. 1(a)]. Afterwards, shear forces, $F_{S}$, were applied quasistatically until motion either spontaneously initiated or was externally triggered [33].

Figure 1(b) presents typical measurements of $A(x, t)$ for both sub-Rayleigh $\left(C_{f}<C_{R}\right)$ and supershear $\left(C_{f}>C_{S}\right)$ fronts where $C_{f}$ is the rupture front velocity. The onset of frictional motion is mediated by cracklike rupture fronts that leave in their wake significantly reduced $A$. The boundary between regions of intact and reduced areas of contact defines the rupture tip position, $x_{\text {tip. }}$ In these examples, ruptures nucleated at $x \approx 0$ and accelerated in the positive $x$ direction. Figure 1(c) describes the corresponding evolution of $A(x, t)$ at $x=110 \mathrm{~mm}$. Temporal measurements of $A(x=110 \mathrm{~mm}, t)$ were converted to a spatial profile $A\left(x-x_{\text {tip }}\right)$ by utilizing $A(x, t)=A\left(x-\int C_{f} d t\right)$ [24]. This method eliminated any spatial variation due to inhomogeneities along the frictional interface. Residual contact areas, $A_{r}$, were measured at times corresponding to $x-x_{\text {tip }}=-35 \mathrm{~mm}$, chosen to be both beyond any dynamic variation of $A$ associated with the rupture tip and prior to the arrival of any reflections, as seen in Fig. 1(b) for $t>2 \mathrm{~ms}$ (top) and $t>0.1 \mathrm{~ms}$ (bottom).

We now consider the slip velocity following the passage of ruptures. Figure 1(d) presents measurements of the material velocity, $\dot{u}_{x}$ (where $u_{x}$ is the $x$ component of the displacement field in the lab frame), at $y \approx 3.5 \mathrm{~mm}$ (top plate). As for $A$, temporal measurements of $\dot{u}_{x}$ are converted to spatial profiles. Material velocities, as well as all components of stress and strain fields near the rupture tip $\left(C_{f}<C_{R}\right)$, are dominated by the universal square-root singular form originally developed to describe brittle shear cracks [24,27]. Figure 1(d), top demonstrates the agreement between brittle fracture theory and measured $\dot{u}_{x}(y=3.5 \mathrm{~mm}$ ) (solid black line). For $x-x_{\text {tip }}<-20 \mathrm{~mm}$ measured $\dot{u}_{x}(y=3.5 \mathrm{~mm})$ reliably reflect the velocities at the interface $\dot{u}_{x}\left(y=0^{+}\right)$. The relative local velocity between the plates, the slip velocity, is given by $v=2 \dot{u}_{x}\left(y=0^{+}\right)$as $\dot{u}_{x}(x, y)$ is antisymmetric with respect to $y$ [34]. (In the case of the thin top plate on the thick bottom block, where antisymmetry does not strictly apply, we expect only small corrections to $v$ for $C_{f}<0.9 C_{R}$ [35].) Faster ruptures are associated with larger $v$ [Fig. 1(d)] and are accompanied by lower values of $A_{r}$ [Fig. 1(c)]. In what follows, local values of $v$ (measured simultaneously with $A_{r}$ ) will be systematically related to $A_{r}$ and frictional resistance at the tails of propagating rupture fronts.

Figure 2(a) presents measurements of $\varepsilon_{x y}$ at $y \approx 3.5 \mathrm{~mm}$ for the two rupture events presented in Fig. 1. In order to calculate the shear stresses, $\sigma_{x y}$, the viscoelastic constitutive law for PMMA [36,37] must be used. For a viscoelastic material in the region of linear response at small strains $\sigma_{x y}(\omega)=2 \mu^{*}(\omega) \varepsilon_{x y}(\omega)$, where $\mu^{*}(\omega)$ is the frequency dependent complex shear modulus. Its real and imaginary parts are the storage and loss modulus, respectively. In our case, we neglect the loss modulus [36]. We further simplify the analysis by using the large separation of time scales between the long interevent time $(\sim 10 \mathrm{~s})$ and the short times $(10-500 \mu \mathrm{s})$ associated with the rupture passage. This yields

$$
\sigma_{x y}(t)=2 \mu_{f}\left[\varepsilon_{x y}(t)-\varepsilon_{x y}^{0}\right]+2 \mu_{s} \varepsilon_{x y}^{0},
$$

where $\mu_{f}=2.1 \pm 3 \%$ and $\mu_{s}=1.2 \pm 5 \%$ GPa are directly measured fast and slow storage moduli and $\varepsilon_{x y}^{0}$ are initially imposed shear strains, as denoted in Fig. 2(a). The validity of this approximation was explicitly verified. The calculated $\sigma_{x y}$ are plotted in Fig. 2(b). Figure 2 demonstrates that it is important to properly account for viscoelasticity.

Residual stresses, $\tau_{r}$, are measured at times corresponding to $x-x_{\text {tip }}=-35 \mathrm{~mm}$, which are well beyond dynamic 

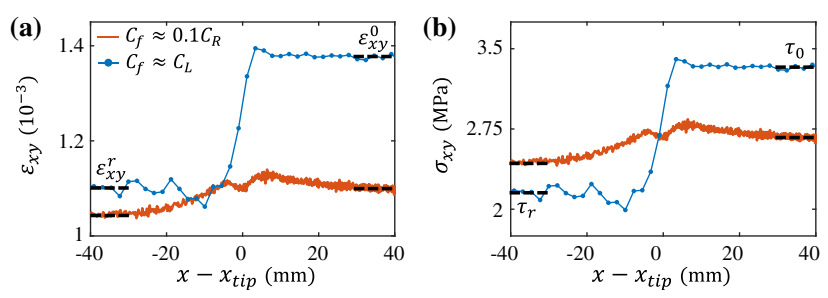

FIG. 2. Stress calculations are highly influenced by properly accounting for viscoelasticity. (a) Examples of the measured $\varepsilon_{x y}$ plotted relative to the rupture tip position $x_{\text {tip }}=110 \mathrm{~mm}$. $\varepsilon_{x y}^{0}$ is the initial strain level, prior to the rupture arrival. (b) Shear stresses, $\sigma_{x y}$, are calculated from the measured $\varepsilon_{x y}$ by using the viscoelastic constitutive law of PMMA [Eq. (1)]. $\tau_{r}$, the residual frictional resistance are measured at times corresponding to $x-x_{\text {tip }}=-35 \mathrm{~mm}$ beyond any dynamic stress variations associated with the rupture tip. Note that the decrease of $\tau_{r}$ with $C_{f}$ only becomes apparent when viscoelasticity is accounted for. (a), (b) Profiles of $\varepsilon_{x y}$ and $\sigma_{x y}$ corresponding to the $A / A_{0}$ measurements in Fig. 1(c) during slow (orange line) and supershear (blue line) rupture events. Local $\sigma_{y y}$ values are the same for both rupture events.

stress variations associated with the rupture tip. $\tau_{r}$, therefore, represent the local frictional resistance during sliding due to the nonzero value of $A_{r}$ after the rupture passage [Fig. 1(c)]. To first order the values of $\tau_{r}$ scale linearly with the local normal stress, $\sigma_{y y}(y=0)$, denoted from here on by $\sigma$ [Fig. S1(a)], where the proportionality factor is the local dynamic friction coefficient. For a given $\sigma$, however, $\tau_{r}$ is not constant [Fig. 2(b)]. Here we focus on the (second order) rate dependence of $\tau_{r}$. The difference $\Delta \tau=\tau_{0}-\tau_{r}$ provides a measure of the amount of released elastic energy and plays a key role in both rupture dynamics [33] and rupture arrest [38,39].
In Fig. 3 we consider the systematic variations of $\tau_{r}$ and $A_{r}$ with simultaneously measured slip velocities, $v$. The figure is composed of multiple spatial measurements within $\sim 30$ rupture events over a wide range of $C_{f} ; \sim 0.05 C_{R}<$ $C_{f}<\sim C_{R}$ (orange and green symbols) and $C_{f} \approx C_{L}$ (blue symbols). In this range of $C_{f}, v$ spanned over 2 orders of magnitude. Note that for the experimental setup of a thin top plate on a thick bottom block at $C_{f} \rightarrow C_{R}$ geometrical effects result in a coupling between $v$ and $\sigma$ [35]. Therefore, in this block geometry (green symbols) only $C_{f}<0.9 C_{R}$ were considered.

The measurements in Fig. 3(a) and 3(b) reveal the existence of a critical slip velocity, $v_{c}(\sim 0.1 \mathrm{~m} / \mathrm{s})$, that signals a transition in the frictional behavior. For $v<v_{c}$, $A_{r}$ decreases logarithmically with $v$ while no significant change is observed in $\tau_{r}$. For $v>v_{c}$, the rate of logarithmic decrease of $A_{r}$ becomes 2.5 times larger and velocity weakening of $\tau_{r}$ commences. If the frictional properties of the interface $\left(\tau_{r}\right.$ and $\left.A_{r}\right)$ were solely dependent on the instantaneous slip velocity, then we would expect that our measurements for sub-Rayleigh (orange symbols) and supershear (blue symbols) ruptures would collapse onto the same curves. While this is approximately true, differences in the two rupture regimes are apparent in both $\tau_{r}$ and $A_{r}$ in Fig. 3. These differences suggest a nontrivial history dependence of the frictional properties of the interface; $\tau_{r}$ and $A_{r}$ are not solely dependent on instantaneous values of $v$. One would not expect this history dependence if contacts were wholly renewed during slip.

Several comments are in order. First, $\sim 20 \%$ spatial variations of $\tau_{r} / \sigma$ (Fig. S1) are common. In Fig. 3(a) we accounted for these spatial variations by normalizing the measurements at different locations (See Sec. I in Ref. [28]). Second, the $\sim 5 \%$ uncertainty in $\mu_{f}$ and $\mu_{s}$ values
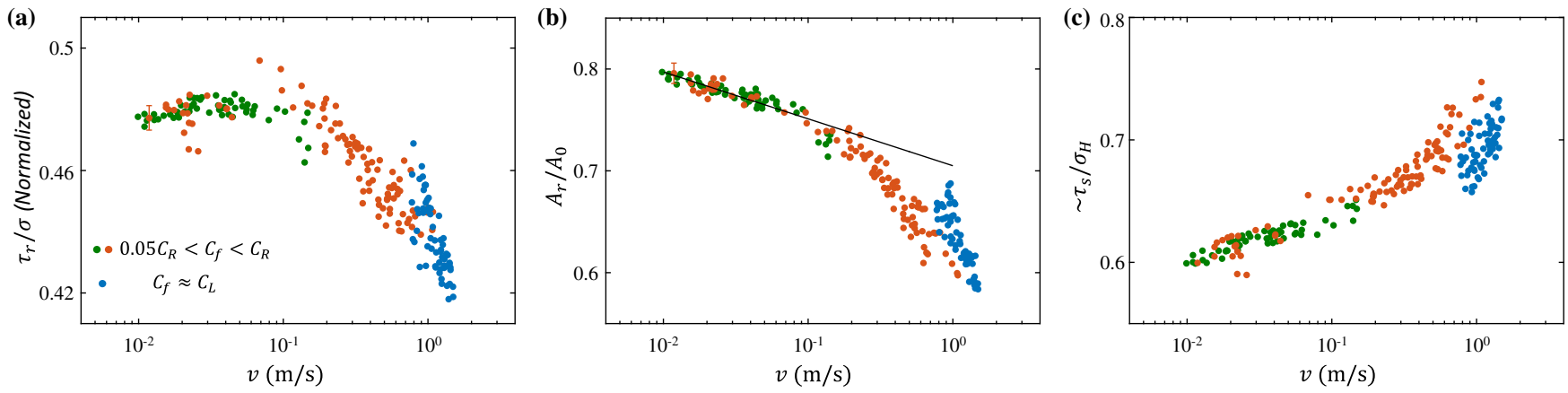

FIG. 3. Enhanced velocity weakening above a critical slip velocity, $v_{c}$. (a) Variation of the residual frictional resistance, $\tau_{r}$ [Fig. 2(b)], as a function of the local slip velocity [Fig. 1(d)]. $\tau_{r}$ weakens with increasing $v$ for $v>v_{c}(\sim 0.1 \mathrm{~m} / \mathrm{s})$. Values of $\tau_{r} / \sigma$ were normalized to account for spatial variations of the measurements (see Sec. I in Ref. [28]). (b) Corresponding measurements of the residual contact area, $A_{r} / A_{0}$ [Fig. 1(c)]. The rate of the logarithmic reduction of $A_{r}$ with $v$ increases by a factor of $\sim 2.5$ at $v>v_{c}$. This enhanced reduction of $A_{r}$ is concurrent with the weakening of $\tau_{r}$. (c) The rate dependence of the contact shear strength, $\tau_{s}$, is obtained by taking the ratio of $\tau_{r} / \sigma$ and $A_{r} / A_{0}$. This ratio roughly scales as $\tau_{s} / \sigma_{H}$, where $\sigma_{H}$ is the material hardness. (a)-(c) Green is the thin top plate on thick bottom block. In this block geometry only $C_{f}<0.9 C_{R}$ were considered. Orange $\left(\sim 0.05 C_{R}<C_{f}<\sim C_{R}\right)$ and blue $\left(C_{f} \approx C_{L}\right)$ correspond to identical geometries of the top and bottom plates; $\sim 30$ rupture events are presented. The measurements are all local measurements performed in the central region $90 \mathrm{~mm}<x<140 \mathrm{~mm}$ of our interfaces. 
does not affect the presented rate dependence of $\tau_{r}$. Third, we have explicitly verified that interevent healing times (5-15 s) result in very small variations of $A_{0}(<5 \%)$; therefore, the rate dependence of $A_{r} / A_{0}$ presented in Fig. 3(b) should be attributed to the rate dependence of $A_{r}$.

$A$, the local sum of the microsize contacts, is significantly smaller than the nominal contact area, $A_{n}$ [40]. In the continuum limit, $\tau$, the local frictional resistance (stress) is expected to be [1]

$$
\tau=A / A_{n} \tau_{s},
$$

where $\tau_{s}$ is the shear strength per unit area of the contacts. Using Eq. (2) and the relation $A_{0} / A_{n} \sim \sigma / \sigma_{H}$, where $\sigma_{H}$ is the material hardness [1,2], therefore, enables us to determine $\tau_{s}$ during sliding from direct measurements of $\tau_{r} / \sigma$ and $A_{r} / A_{0}$ by $\tau_{s} / \sigma_{H} \sim\left(\tau_{r} / \sigma\right) /\left(A_{r} / A_{0}\right)$. Figure 3(c) presents the rate dependence of $\tau_{s}$ and shows that $\tau_{s}$ continuously increases with $v$, despite the decreasing values of both $\tau_{r}$ and $A_{r}$ with $v$.

We now consider the rate dependence of $A$. Generally, $A$ exhibits nontrivial rate and history dependence which are not yet completely understood. In the rate and state framework [3], $A$ is often described by

$$
A / A_{n}=\sigma / \sigma_{H}\left[1+\beta \log \left(1+\phi / \phi^{*}\right)\right],
$$

where $\phi$ is a state variable $[3,4,6,11,41-43]$ that is typically interpreted as the "contact lifetime" and $\phi^{*}$ is a short time cutoff $[3,12,44]$. Setting $\phi=t$, for instance, captures the logarithmic increase of $A$ with the contact time (aging) of two pressed materials $[23,40]$ (Fig. S2). Furthermore, logarithmic velocity weakening of $\tau$, observed in experiments with imposed slow slip velocities $\left(v<10^{2} \mu \mathrm{m} / \mathrm{s}\right)$ [3-5], has been explained via Eq. (2) with the suggestion that $A$ decreases due to the reduction of the contact lifetime with increasing $v ; \phi \sim d_{c} / v$, where $d_{c}$ is a typical contact size.

Figure 3(b) provides direct evidence for such a logarithmic decrease of $A$ for local values of $v$ at slow rates $\left(<v_{c}\right)$. For $v<v_{c}$ we estimate $\beta$ as $0.03 \pm 0.01$ (Sec. II in Ref. [28]). This value of $\beta$, while consistent with $\beta$ measured during the aging of $A$ (Fig. S2), is $20 \%-80 \%$ smaller. This observation contrasts with experiments on rock [6], where the values of $\beta$ measured during aging and sliding by sound transmission across the interface were more closely comparable. Our experiments significantly differ from sliding experiments in rock, as here the slip rates are both much higher, measurements are local, and no powderlike ("gouge") layer of ground rock is formed. Our observations may suggest that the form of Eq. (3) may be too simple.

What is the origin of the transition at $v_{c}$ ? Rapid temperature increase (flash heating) is expected during the contact lifetime due to frictional heating at high slip rates [14]. By taking into account the generated heat $\left(\tau_{s} v\right)$ and the heat transport away from the interface to the material bulk, this temperature increase can be estimated. These calculations imply that contact temperatures should reach the glass $(\sim 400 \mathrm{~K})$ temperatures of PMMA at slip rates in the range of $0.1-1 \mathrm{~m} / \mathrm{s}$ (Fig. S3), suggesting significant contact softening. This transitional slip rate is indeed consistent with the observed transition velocity, $v_{c} \sim 0.1 \mathrm{~m} / \mathrm{s}$. In macroscopic sliding experiments where bulk PMMA blocks were externally heated to near their glass temperature, decreased friction was indeed observed [5]. On the other hand, we would intuitively expect that such severe softening of the contacts should be accompanied by large reductions in both $\tau_{r}$ and $\tau_{s}$. This, remarkably, is not evident in the data; our observed reduction of $\tau_{r}$ is only at the level of a few percent. This highly nonintuitive result is echoed in the inferred frictional strength of the contacts $\tau_{s}$, which continuously increases with $v$ [Fig. 3(c)]. This increase of $\tau_{s}$ takes place, in contrast to $\tau_{r}$, both below and above $v_{c}$. While logarithmic velocity strengthening of $\tau_{s}$ for $v<v_{c}$ is generally interpreted as thermally activated creep [3,7-10], the increase of $\tau_{s}$ with $v$ above $v_{c}$, where softening of the contacts is expected, could be the result of competition between temperature-induced weakening and rate-induced strengthening [10] of material properties (e.g., yield stress [45]).

The mild decrease of $\tau_{r}(\sim 10 \%)$ reported here is in stark contrast to both recent rupture experiments in Homalite [26] and to rock friction experiments [15-18], where $v$ is imposed. These experiments demonstrated a significant reduction of the friction coefficient (up to an order of magnitude) that was attributed to the same flash heating mechanism. One significant difference with our experiments is that the experiments in rock form a continuous gouge layer of ground rock that is sandwiched between the sliding surfaces. In our experiments no gouge is formed and the interface is always composed of a sparse ensemble of discrete contacts.

Understanding these results is a challenge that could be related to the qualitative character of frictional interfaces. Our experiments present strong evidence that, while flash heating may occur, this mechanism is not, in itself, a necessary condition for strong frictional weakening. The answers could lie in better understanding the complex dynamics of discrete contacts under extreme conditions and slip rates.

We thank E. Bouchbinder, Y. Bar-Sinai, and M. Aldam for fruitful comments. We also acknowledge the support of both the US-Israel Binational Science Foundation (Grant No. 2016950), and Israel Science Foundation (Grant No. 1523/15). E. B. acknowledges the support from the Lady Davis Trust.

[1] F. P. Bowden and D. Tabor, The Friction and Lubrication of Solids, 2nd ed. (Oxford Univ. Press, New York, 2001).

[2] J. H. Dieterich and B. D. Kilgore, Tectonophysics 256, 219 (1996). 
[3] T. Baumberger and C. Caroli, Adv. Phys. 55, 279 (2006).

[4] C. Marone, Annu. Rev. Earth Planetary Sci. 26, 643 (1998).

[5] T. Baumberger, P. Berthoud, and C. Caroli, Phys. Rev. B 60, 3928 (1999).

[6] K. Nagata, M. Nakatani, and S. Yoshida, J. Geophys. Res. 117, B02314 (2012).

[7] M. Nakatani, J. Geophys. Res. 106, 13347 (2001).

[8] J. R. Rice, N. Lapusta, and K. Ranjith, J. Mech. Phys. Solids 49, 1865 (2001).

[9] T. Putelat, J. H. Dawes, and J. R. Willis, J. Mech. Phys. Solids 59, 1062 (2011).

[10] Y. Bar-Sinai, R. Spatschek, E. A. Brener, and E. Bouchbinder, J. Geophys. Res. 119, 1738 (2014).

[11] B. M. Kaproth and C. Marone, Science 341, 1229 (2013).

[12] Y. Bar-Sinai, R. Spatschek, E. A. Brener, and E. Bouchbinder, Phys. Rev. E 88, 060403 (2013).

[13] J. C. Chang, D. A. Lockner, and Z. Reches, Science 338, 101 (2012).

[14] J. R. Rice, J. Geophys. Res. 111 (2006).

[15] D. L. Goldsby and T. E. Tullis, Science 334, 216 (2011).

[16] G. Di Toro, R. Han, T. Hirose, N. De Paola, S. Nielsen, K. Mizoguchi, F. Ferri, M. Cocco, and T. Shimamoto, Nature (London) 471, 494 (2011).

[17] K. M. Brown and Y. Fialko, Nature (London) 488, 638 (2012).

[18] F. X. Passelègue, D. L. Goldsby, and O. Fabbri, Geophys. Res. Lett. 41, 828 (2014).

[19] S. M. Rubinstein, G. Cohen, and J. Fineberg, Nature (London) 430, 1005 (2004).

[20] P. G. Okubo and J. H. Dieterich, J. Geophys. Res. 89, 5817 (1984).

[21] M. Ohnaka, The Physics of Rock Failure and Earthquakes, 1st ed. (Cambridge University Press, New York, 2013).

[22] M. Mello, H. S. Bhat, and A. J. Rosakis, J. Mech. Phys. Solids 93, 153 (2016).

[23] O. Ben-David, S. M. Rubinstein, and J. Fineberg, Nature (London) 463, 76 (2010).

[24] I. Svetlizky and J. Fineberg, Nature (London) 509, 205 (2014).

[25] F. X. Passelègue, A. Schubnel, S. Nielsen, H. S. Bhat, D. Deldicque, and R. Madariaga, J. Geophys. Res. 121, 4343 (2016).
[26] V. Rubino, A. Rosakis, and N. Lapusta [Nature Commun. (to be published)].

[27] I. Svetlizky, D. Pino Muñoz, M. Radiguet, D. S. Kammer, J.-F. Molinari, and J. Fineberg, Proc. Natl. Acad. Sci. U.S.A. 113, 542 (2016).

[28] See Supplemental Material at http://link.aps.org/ supplemental/10.1103/PhysRevLett.118.234301 for details of the experimental analysis, measurements of the contact area aging, and estimations of the temperature increase. Included are Refs. [29-31].

[29] O. Ben-David, G. Cohen, and J. Fineberg, Science 330, 211 (2010).

[30] B. J. Briscoe and K. S. Sebastian, Proc. R. Soc. A 452, 439 (1996).

[31] P. Berthoud, T. Baumberger, C. G'Sell, and J.-M. Hiver, Phys. Rev. B 59, 14313 (1999).

[32] S. M. Rubinstein, M. Shay, G. Cohen, and J. Fineberg, Int. J. Fract. 140, 201 (2006).

[33] I. Svetlizky, D. S. Kammer, E. Bayart, G. Cohen, and J. Fineberg, Phys. Rev. Lett. 118, 125501 (2017).

[34] H. Shlomai and J. Fineberg, Nat. Commun. 7, 11787 (2016).

[35] M. Aldam, Y. Bar-Sinai, I. Svetlizky, E. A. Brener, J. Fineberg, and E. Bouchbinder, Phys. Rev. X 6, 041023 (2016).

[36] B. E. Read and J.C. Duncan, Polymer Testing 2, 135 (1981).

[37] M. Radiguet, D. S. Kammer, P. Gillet, and J.-F. Molinari, Phys. Rev. Lett. 111, 164302 (2013).

[38] D. S. Kammer, M. Radiguet, J.-P. Ampuero, and J.-F. Molinari, Tribol. Lett. 57, 23 (2015).

[39] E. Bayart, I. Svetlizky, and J. Fineberg, Nat. Phys. 12, 166 (2016).

[40] J. H. Dieterich and B. D. Kilgore, Pure Appl. Geophys. 143, 283 (1994).

[41] V. Popov, B. Grzemba, J. Starcevic, and C. Fabry, Physical Mesomechanics 13, 283 (2010).

[42] P. Bhattacharya, A. M. Rubin, E. Bayart, H. M. Savage, and C. Marone, J. Geophys. Res. 120, 6365 (2015).

[43] A. Cabboi, T. Putelat, and J. Woodhouse, J. Mech. Phys. Solids 92, 210 (2016).

[44] M. Nakatani and C. H. Scholz, J. Geophys. Res. 111 (2006).

[45] J. Richeton, S. Ahzi, K. Vecchio, F. Jiang, and R. Adharapurapu, Int. J. Solids Struct. 43, 2318 (2006). 